\section{A glenlda}

\section{Möchten Sie hier einen Kongress anzeigen?}

Bitte schicken Sie vollständige Angaben (genaues Datum, Ort, Titel der Veranstaltung, Kontaktadresse einschliesslich E-Mail) für diese Rubrik an: dermatologica.helvetica@hcuge.ch. Danke.

\section{Voudriez-vous annoncer un congrès?}

Veuillez envoyer, s.v.p., les spécifications complètes (date exacte, lieu, thème du congrès, adresse de contact avec E-mail) pour cette rubrique à: dermatologica.helvetica@hcuge.ch.

\section{In der Schweiz/En Suisse}

Woche $4 / 4$ e semaine

23.1.2003

Jahrestagung der Gesellschaft für Phlebologie, Basel

\section{Réunion annuelle de la Société Suisse de}

\section{Phlébologie, Bâle}

Hörsaal, Klinikum I, Spitalstrasse 21

«Thromboembolie Update 2003»

Wiss. Organisatoren Prof. K.A. Jäger, Dr. C. Jeanneret

Sekretariat: Frau H. Baur

Tel. +41612655157

Fax +4161265 53

E-mail christina.jeanneret@ksbh.ch

\section{Woche $23 / 23$ e semaine}

5.6 .2003

Colloque de printemps SSDV/Frühjahrskolloquium SGDV, Genève

«Traitement du psoriasis 2003: présent et futur»

Xavier Didierjean

Hôpital Cantonal Universitaire de Genève

Clinique et Policlinique de Dermatologie

42, rue Micheli-du-Cresl

1211 Genève 14

E-mail dermatologica.helvetica@hcuge.ch

\section{Woche $24 / 24$ e semaine}

Frühjahrskongress der SGP/Réunion de printemps de la SSP, Lausanne

«Perforantes: relevance clinique et traitement»/

«Perforansvenen: Klinische Relevanz und therapeutische Aspekte»

Lausanne, Musée Olympique

Information: www.uvs.ch

Woche $38 / 38$ e semaine

18.9.-20.9.2003

85e Assemblé annuelle SSDV/

85. Jahresversammlung der SGDV, Lausanne

Sujet principal: «Dermato-oncologie»

Hauptthema: «Dermatoonkologie»

Info: Prof. D. Hohl/Mme G. Maradan

Fax 0213140382

E-mail congressdv2003@hospvd.ch
Im Ausland/A I'étranger

Week 12/13

March 21-26, 2003

61st American Academy of Dermatology Annual Meeting, San Francisco

Info: American Academy of Dermatology

Department of Meetings and Conventions

PO Box 4014

Schaumburg IL 60168-4014

Fax +18473301090

www.aad.org

Woche $18 / 18$ e semaine

30.4.-4.5. 2003

24e Congrès International de l'Association des Dermatologistes Francophones, Sfax, Tunisie

Président du congrès: Prof. Abdelmajid Zahaf abdelmajid@zahaf.net

Information et Bulletin d'inscription: www.dermato-tunisie.com

Week 18

April 30-May 5, 2003

Joint Meeting of the ESDR, SID and JSID, Miami Beach

«International Investigative Dermatology 2003»

Info: Society for Investigative Dermatology

Suite 340

820 West Superior Avenue

Cleveland, $\mathrm{OH}$ 44113-1800

Tel. +1 2165799300

Fax +12165799333

sid@sidnet.org

www.sidnet.org

\section{Woche 19}

6.-10.5.2003

42. Jahrestagung der Deutschen Dermatologischen Gesellschaft, Berlin

Info: www.derma.de

Week 42

October 15-18, 2003

European Academy of Dermatology Annual

Congress, Barcelona

Info: General Secretariat Unicongress

Calvet, 55

08021 Barcelona (Spain)

Tel. + 34932007088

Fax + 34934146817

eadv2003@unicongress.com 